Fábio Jessen Werneck de Almeida Martins

\title{
Métodos de Aceleração de Aprendizado Aplicado ao Modelo Neuro-Fuzzy Hierárquico Politree com Aprendizado por Reforço
}

Dissertação apresentada ao Programa de Pósgraduação em Engenharia Elétrica da PUC-Rio como requisito parcial para obtenção do título de Mestre em Engenharia Elétrica.

Orientadora: Prof. a Marley Vellasco

Co-orientadora: Prof. ${ }^{\text {a }}$ Karla Figueiredo 


$$
\text { Pontifícia Universidade } \text { Católica }_{\text {do Rio de Janeiro }}
$$

Fábio Jessen Werneck de Almeida Martins

\begin{abstract}
Métodos de Aceleração de Aprendizado Aplicado ao Modelo Neuro-Fuzzy Hierarquico Politree com Aprendizado por Reforço
\end{abstract}

Dissertação de Mestrado apresentada como requisito parcial para obtenção do grau de Mestre pelo Programa de Pós-Graduação em Engenharia Elétrica do Departamento de Engenharia Elétrica do Centro Técnico Científico da PUC-Rio. Aprovada pela Comissão Examinadora abaixo assinada.

Profa. Marley Maria Bernardes Rebuzzi Vellasco Orientadora Departamento de Engenharia Elétrica - PUC-Rio

Profa. Karla Tereza Figueiredo Leite Co-Orientadora Departamento de Engenharia Elétrica - PUC-Rio

Prof. Ricardo Tanscheit Departamento de Engenharia Elétrica - PUC-Rio

Prof. Flávio Joaquim de Souza

UERJ

Prof. Reinaldo Augusto da Costa

Bianchi

Centro Universitário da FEI

Prof. Adriano Joaquim de Oliveira

Cruz

NCE

Prof. José Eugenio Leal Coordenador Setorial do Centro

Técnico Científico - PUC-Rio

Rio de Janeiro, 12 de julho de 2010 
Todos os direitos reservados. É proibida a reprodução total ou parcial do trabalho sem autorização da universidade, do autor e da orientadora.

Fábio Jessen Werneck de Almeida Martins

Graduou-se em Engenharia Mecânica na Pontifícia Universidade Católica do Rio de Janeiro (PUC-Rio) em 2007. Graduou-se em Engenharia de Controle e Automação na PUC-Rio.

Ficha Catalográfica

Martins, Fábio Jessen Werneck de Almeida

Métodos de aceleração de aprendizagem aplicado ao modelo neuro-fuzzy hierárquico politree com aprendizado por reforço / Fábio Jessen Werneck de Almeida Martins orientadora: Marley Vellasco ; coorientadora: Karla Figueiredo. - 2010.

157 f. : il. ; $30 \mathrm{~cm}$

Dissertação (mestrado)-Pontifícia Universidade Católica do Rio de Janeiro, 2010.

Inclui bibliografia

1. Engenharia elétrica - Teses. 2. Agente inteligente. 3. Neuro-fuzzy. 4. Aprendizado por reforço. 5. Aprendizado automático. 6. Modelo hierárquico politree. I. Vellasco, Marley. II. Figueiredo, Karla. III. Pontifícia Universidade Católica do Rio de Janeiro. Departamento de Engenharia Elétrica. IV. Título.

CDD: 621.3 
Para meus pais, Manuel e Magali, pelo apoio e confiança. 


\section{Agradecimentos}

Às minhas orientadoras Professora Marley Vellasco e Professora Karla Figueiredo pelo ensinamentos, estímulo e parceria para a realização deste trabalho.

Ao CNPq, Faperj e à PUC-Rio, pelos auxílios concedidos, sem os quais este trabalho não poderia ter sido realizado.

Aos meus pais, pela educação, atenção e carinho.

À minha madrinha, com seus 94 anos de otimismo, por suas palavras de apoio.

Ao meu irmão, pela companhia de todas as horas.

Aos meus amigos e amigas por todo apoio, paciência e compreensão.

Aos meus colegas da PUC-Rio.

Aos professores que participaram da Comissão examinadora.

A todos os professores e funcionários da faculdade que de uma forma ou de outra me ajudaram. 


\section{Resumo}

Martins, Fábio Jessen Werneck de Almeida; Vellasco, Marley (Orientadora); Figueiredo, Karla (Orientadora). Métodos de Aceleração de Aprendizado Aplicado ao Modelo Neuro-Fuzzy Hierárquico Politree com Aprendizado por Reforço. Rio de Janeiro, 2010. 157p. Dissertação de Mestrado - Departamento de Engenharia Elétrica, Pontifícia Universidade Católica do Rio de Janeiro.

Neste trabalho foram desenvolvidos e avaliados métodos com o objetivo de melhorar e acelerar o processo de aprendizado do modelo de Reinforcement Learning Neuro-Fuzzy Hierárquico Politree (RL-NFHP). Este modelo pode ser utilizado para dotar um agente de inteligência através de processo de Aprendizado por Reforço (Reinforcement Learning). O modelo RL-NFHP apresenta as seguintes características: aprendizado automático da estrutura do modelo; auto-ajuste dos parâmetros associados à estrutura; capacidade de aprendizado da ação a ser adotada quando o agente está em um determinado estado do ambiente; possibilidade de lidar com um número maior de entradas do que os sistemas neuro-fuzzy tradicionais; e geração de regras linguísticas com hierarquia. Com intenção de melhorar e acelerar o processo de aprendizado do modelo foram implementadas seis políticas de seleção, sendo uma delas uma inovação deste trabalho (Q-DC-roulette); implementado o método early stopping para determinação automática do fim do treinamento; desenvolvido o eligibility trace cumulativo; criado um método de poda da estrutura, para eliminação de células desnecessárias; além da reescrita do código computacional original. O modelo RL-NFHP modificado foi avaliado em três aplicações: o benchmark Carro na Montanha simulado, conhecido na área de agentes autônomos; uma simulação robótica baseada no robô Khepera; e uma num robô real NXT. Os testes efetuados demonstram que este modelo modificado se ajustou bem a problemas de sistemas de controle e robótica, apresentando boa generalização. Comparado o modelo RL-NFHP modificado com o original, houve aceleração do aprendizado e obtenção de menores modelos treinados.

\section{Palavras-chave}

Agente Inteligente; Neuro-Fuzzy; Aprendizado por Reforço; Aprendizado Automático; Modelo Hierárquico Politree 


\section{Abstract}

Martins, Fábio Jessen Werneck de Almeida; Vellasco, Marley (Advisor); Figueiredo, Karla (Advisor). Methods for Acceleration of Learning Process of Reinforcement Learning Neuro-Fuzzy Hierarchical Politree Model. Rio de Janeiro, 2010. 157p. MSc. Dissertation - Departamento de Engenharia Elétrica, Pontifícia Universidade Católica do Rio de Janeiro.

In this work, methods were developed and evaluated in order to improve and accelerate the learning process of Reinforcement Learning Neuro-Fuzzy Hierarchical Politree Model (RL-NFHP). This model is employed to provide an agent with intelligence, making it autonomous, due to the capacity of ratiocinate (infer actions) and learning, acquired knowledge through interaction with the environment by Reinforcement Learning process. The RL-NFHP model has the following features: automatic learning of structure of the model; self-adjustment of parameters associated with its structure, ability to learn the action to be taken when the agent is in a particular state of the environment; ability to handle a larger number of inputs than the traditional neuro-fuzzy systems; and generation of rules with linguistic interpretable hierarchy. With the aim to improve and accelerate the learning process of the model, six selection action policies were developed, one of them an innovation of this work ( $Q$-DC-roulette); implemented the early stopping method for automatically determining the end of the training; developed a cumulative eligibility trace; created a method of pruning the structure, for removing unnecessary cells; in addition to rewriting the original computer code. The modified RL-NFHP model was evaluated in three applications: the simulated benchmark Car-Mountain problem, well known in the area of autonomous agents; a simulated application in robotics based on the Khepera robot; and an application in a real robot. The experiments show that this modified model fits well the problems of control systems and robotics, with a good generalization. Compared the modified RL-NFHP model with the original one, there was acceleration of learning process and smaller structures of the model trained.

\section{Keywords}

Intelligent Agent; Neuro-Fuzzy Systems; Learning; Reinforcement Learning; Automatic Learning; Hierarchical Politree Model 


\section{Sumário}

1. INTRODUÇÃO 17

1.1. Motivação 17

1.2. Objetivo 20

1.3. Descrição do Trabalho e Contribuições 20

1.4. Estrutura do Trabalho $\quad 22$

2. MODELO DE REINFORCEMENTE LEARNING NEURO-FUZZY HIERÁRQUICO POLITREE (RL-NFHP) 23

2.1. Introdução 23

2.2. Particionamento Politree 25

2.3. Célula Básica RL-Neuro-Fuzzy Hierárquico Politree 26

2.4. Arquitetura RL-NFHP 30

2.4.1. Antecedentes das Regras do Modelo RL-NFHP 34

2.4.2. Consequentes das Regras do Modelo RL-NFHP 35

2.5. Algoritmo de Aprendizado 37

2.5.1. Criação do Controle RL-NFHP 37

2.5.2. Início da Simulação 39

2.5.3. Seleção das Ações $\quad 39$

2.5.4. Cálculo da Saída $\quad 40$

2.5.5. Cálculo do Retorno e Retropropagação 44

2.5.6. Atualização da Função Valor Q 45

2.5.7. Particionamento $\quad 50$

2.5.8. Fim do Episódio 56

2.5.9. Fim do Treinamento 56

2.6. Teste e Uso do Modelo RL-NFHP

3. MÉTODOS DE ACELERAÇÃO DO MODELO RL-NFHP 58

3.1. Políticas de seleção de ação $\quad 58$

3.1.1. Política $\varepsilon$-greedy 58

3.1.2. Política $Q$-roulette $\quad 59$

3.1.3. Política $D C$-roulette 60

3.1.3. Política $Q+D C$-roulette 61 
3.1.5. Política $Q-D C$-roulette 61

3.2. Early stopping 64

3.3. Elegibility trace cumulativo 64

3.4. Poda da estrutura RL-NFHP 66

3.5. Reescrita do algoritmo 66

4. ESTUDO DE CASOS 70

4.1. Carro na montanha (Mountain-Car Problem) 70

4.1.1. Experimento 1: Política de escolha das ações 73

4.1.2. Experimento 2: Variação da probabilidade de escolha aleatória 83

4.1.3. Experimento 3: Função de crescimento 86

4.1.4. Experimento 4: Elegibility Trace Cumulativo 90

4.1.5. Experimento 5: Alfa de atualização de Q(s,a) 94

4.1.6. Experimento 6: Função de Atualização 98

4.1.7. Experimento 7: Comparação entre modelos 103

4.2. Aplicação simulada em robótica (Khepera) 113

4.2.1. Experimento 1: Sem obstáculo 117

4.2.2. Experimento 2: Poda da estrutura 121

4.2.3. Experimento 3: Com obstáculo 123

4.3. Aplicação real em robótica (Lego MindStorms NXT) 131

5. CONCLUSÕES 140

5.1. Conclusões 140

5.2. Sugestões para Trabalhos Futuros 143

6. REFERÊNCIAS BIBLIOGRÁFICAS 145

APÊNDICE: APRENDIZADO POR REFORÇO 149 


\section{Lista de Figuras}

Figura 2.1: (a) Exemplo de particionamento Politree para espaços

de dimensão 2 e (b) árvore representativa deste particionamento.

Figura 2.2: Célula Reinforcement Learning Neuro-Fuzzy

Quadtree (Politree com $n=2$ ).

Figura 2.3: Exemplo de perfil das funções de pertinência da célula RL-NFP.

Figura 2.4: Célula RL-NFP representada sob o formato

de rede neuro-fuzzy.

Figura 2.5: Divisão em quadrantes realizada pelas FPs alto e baixo com entrada incidindo sobre o quadrante 4 .

Figura 2.6: (a) Exemplo de arquitetura RL-NFHP e

(b) Representação genérica em árvore do modelo

RL-NFHP com n entradas e três células (círculos), onde cada célula possui $\mathrm{m}=2^{\mathrm{n}}$ consequentes (quadrados).

Figura 2.7: Esquema do processo de aprendizado do agente

Figura 2.8: Interior da célula RL-NFHP com duas entradas (Quadtree).

Figura 2.9: Fluxograma de treinamento.

Figura 2.10: (a) Exemplo de arquitetura RL-NFHP com 2 entradas

e (b) sua representação em árvore. Esta estrutura possui duas células (círculos), onde cada célula tem 4 consequentes (quadrados).

Figura 2.11: Exemplo de arquitetura RL-NFHP de 2 entradas, mostrando os graus de pertinência dos conjuntos fuzzy das duas células que compõe a estrutura.

Figura 2.12: Exemplo de arquitetura RL-NFHP de 2 entradas, mostrando os graus de pertinência dos conjuntos fuzzy das duas células que compõe a estrutura.

Figura 2.13: (a) Exemplo de arquitetura RL-NFHP com 2 entradas e (b) sua representação em árvore mostrando a retropropagação do retorno do ambiente. Esta estrutura possui duas células (círculos), onde cada célula tem 4 consequentes (quadrados).

Figura 2.14: Caso exemplo de atualização da função de valor Qt+1 relativa à eq. 2.17.

Figura 2.15: Particionamento da célula RL-NFP 0 .

Figura 2.16: Função de crescimento. 
Figura 3.1: Probabilidade de seleção das ações da tabela 3.1

de acordo com as políticas: (a) Q-roulette;

(b) Q-roulette Giggs-Boltzman; (c) DC-roulette e (d) Q-DC-roulette

Figura 3.2: Hierarquia entre as classes do modelo RL-NFHP 68

Figura 4.1: Benchmark Carro na Montanha 71

Figura 4.2: Tela de simulação do Carro na Montanha 72

Figura 4.3: Particionamento do estado no modelo

RL-NFHP modificado de 23 células.

109

Figura 4.4: Árvore de células do modelo RL-NFHP

modificado de 23 células.

Figura 4.5: Ambiente de simulação do robô. 113

Figura 4.6: Detecção de obstáculo. 114

Figura 4.7: Identificação dos ângulos de interesse. 115

Figura 4.8: Ambiente de simulação do robô Khepera sem obstáculo. 117

Figura 4.9: Ambiente de simulação do robô Khepera com obstáculo. 124

Figura 4.10: Caminho percorrido após aprendizado para posições e ângulos iniciais de 1 a 12.

Figura 4.11: Particionamento do estado no modelo RL-NFHP modificado de 238 células.

Figura 4.12: Cenário real mostrando: (a) o robô;

(b) o obstáculo central; (c) a posição final desejada ou objetivo;

(d) as paredes laterais.

Figura 4.13: Robô Lego MindStorms NXT montado para avaliação prática do modelo RL-NFHP, constituído por:

(a) brick NXT; (b) 3 sensores ultrassônicos; (c) 2 servo-motores.

Figura 4.14: Detalhe da estrutura do TriBot do robô.

Figura 4.15: Caminho percorrido pelo robô real para posições e ângulos iniciais de 1 a 12.

Figura 4.16: Caminho percorrido pelo robô real para posições e ângulos iniciais de 1 a 5. 


\section{Lista de Gráficos}

Gráfico 4.1: Aproveitamento do treinamento. 76

Gráfico 4.2: Histograma da média de ciclos 76

Gráfico 4.3: Histograma das épocas de treinamento usando Q-roulette 77

Gráfico 4.4: Histograma das épocas de treinamento $\begin{array}{ll}\text { usando DC-roulette. } & 78\end{array}$

Gráfico 4.5: Histograma das épocas de treinamento $\begin{array}{ll}\text { usando Q+DC-roulette. } & 78\end{array}$

Gráfico 4.6: Histograma das épocas de treinamento usando Q-DC-roulette. $\quad 79$

Gráfico 4.7: Histograma das épocas de treinamento usando $\varepsilon$-greedy. $\quad 79$

Gráfico 4.8: Histograma do número de células do modelo usando Q-roulette. $\quad 80$

Gráfico 4.9: Histograma do número de células do modelo usando DC-roulette.

Gráfico 4.10: Histograma do número de células do modelo usando Q+DC-roulette.

Gráfico 4.11: Histograma do número de células do modelo usando Q-DC-roulette.

Gráfico 4.12: Histograma do número de células do modelo usando $\varepsilon$-greedy.

Gráfico 4.13: Histograma do número máximo de células ativas por episódio usando Q-DC-roulette.

Gráfico 4.14: Histograma das épocas de treinamento usando DC-roulette.

Gráfico 4.15: Histograma das épocas de treinamento usando Q-DC-roulette.

Gráfico 4.16: Histograma das épocas de treinamento usando $\varepsilon$-greedy.

Gráfico 4.17: Histograma das épocas de treinamento usando DC-roulette.

Gráfico 4.18: Histograma das épocas de treinamento usando Q-DC-roulette.

Gráfico 4.19: Histograma das épocas de treinamento usando $\varepsilon$-greedy. 
Gráfico 4.20: Histograma do número de células do modelo usando DC-roulette.

Gráfico 4.21: Histograma do número de células do modelo usando Q-DC-roulette.

Gráfico 4.22: Histograma do número de células do modelo usando $\varepsilon$-greedy.

Gráfico 4.23: Histograma das épocas de treinamento usando DC-roulette.

Gráfico 4.24: Histograma das épocas de treinamento usando Q-DC-roulette.

Gráfico 4.25: Histograma das épocas de treinamento usando $\varepsilon$-greedy.

Gráfico 4.26: Histograma do número de células do modelo usando DC-roulette.

Gráfico 4.27: Histograma do número de células do modelo usando Q-DC-roulette.

Gráfico 4.28: Histograma do número de células do modelo usando $\varepsilon$-greedy.

Gráfico 4.29: Histograma das épocas de treinamento usando DC-roulette.

Gráfico 4.30: Histograma das épocas de treinamento usando Q-DC-roulette.

Gráfico 4.31: Histograma das épocas de treinamento usando $\varepsilon$-greedy.

Gráfico 4.32: Histograma do número de células do modelo usando DC-roulette.

Gráfico 4.33: Histograma do número de células do modelo usando Q-DC-roulette.

Gráfico 4.34: Histograma do número de células do modelo usando $\varepsilon$-greedy

Gráfico 4.35: Histograma das épocas de treinamento usando DC-roulette.

Gráfico 4.36: Histograma das épocas de treinamento usando Q-DC-roulette.

Gráfico 4.37: Histograma das épocas de treinamento usando $\varepsilon$-greedy.

Gráfico 4.38: Histograma do número de células do modelo usando DC-Roulette. 
Gráfico 4.39: Histograma do número de células do modelo usando Q-DC-Roulette.

Gráfico 4.40: Histograma do número de células do modelo usando $\varepsilon$-greedy.

Gráfico 4.41: Posição e velocidade do carro ao longo do episódio iniciando no vale.

Gráfico 4.42: Posição e velocidade do carro ao longo do episódio iniciando junto à parede.

Gráfico 4.43: Histograma das épocas de treinamento.

Gráfico 4.44: Histograma do número de células do modelo.

Gráfico 4.45: Histograma do número máximo de células ativas por episódio durante a fase de testes.

Gráfico 4.45: Histograma das épocas de treinamento usando Q-DC-roulette com e sem o método de poda.

Gráfico 4.46: Histograma do número de células do modelo usando Q-DC-roulette com e sem o método de poda.

Gráfico 4.47: Distância e ângulo do robô durante um episódio para a condição inicial 8.

Gráfico 4.48: Distância e sensores do robô durante um episódio para a condição inicial 8. 


\section{Lista de Tabelas}

Tabela 3.1: Valores de Q(s,ai) e a quantidade de visitas $\mathrm{C}(\mathrm{s}, \mathrm{ai})$ para determinada polipartição em um estado s.

Tabela 3.2: Arquivos do modelo RL-NFHP. 67

Tabela 4.1: Comparação entre as políticas no modelo RL-NFHP. 82

Tabela 4.2: Comparação entre as taxas de incremento da porcentagem aleatória de $5 \%$ e $40 \%$ para as diferentes políticas no modelo RL-NFHP.

Tabela 4.3: Comparação entre valores de $\mathrm{n}$ da função de crescimento para as diferentes políticas no modelo RL-NFHP.

Tabela 4.4: Comparação entre valores do parâmetro $\alpha$ usado na atualização de Q(s,a) para as diferentes políticas no modelo RL-NFHP. 98

Tabela 4.5: Comparação entre as formas de atualização de Q(s,a), com e sem a equação de punição, para as diferentes políticas no modelo RL-NFHP.

Tabela 4.6: Comparação entre modelos para o benchmark Carro na Montanha.

Tabela 4.7: Comparação entre modelos RL-NFHP para o benchmark Carro na Montanha.

Tabela 4.8: Comparação entre as políticas de seleção no modelo RL-NFHP. 120

Tabela 4.9: Comparação entre o uso ou não do método de poda da estrutura para a política Q-DC-roulette no modelo RL-NFHP. 


\section{Lista de Siglas}

ANFIS: Adaptive-Network-Based Fuzzy Inference System 18

DC-roulette: política de seleção baseada em visita 60

ع-greedy: política de seleção através da maior função valor Q 58

ELF: Evolutionary Logic Fuzzy 19

FACL: Fuzzy-Actor-Critic-Learning 19

$\begin{array}{ll}\text { FP: função de pertinência } & 27\end{array}$

FQL: Fuzzy-Q-Learning 19

FSOM: Fuzzy Self-Organing Map 18

JAFIMA: Java Application Framework for Intelligent and Mobile Agents 144

$\begin{array}{ll}\text { LF: Lógica Fuzzy } & 17\end{array}$

$\begin{array}{lr}\text { NEFCLASS: Neuro-Fuzzy Classification } & 18\end{array}$

$\begin{array}{lr}\text { NEFCON: Neuro-Fuzzy Controller } & 19\end{array}$

$Q+D C$-roulette: política de seleção baseada ora na função valor Q ora na visita 61

Q-DC-roulette: política de seleção baseada na função valor Q mesclada à visita 61

Q-Learning: algoritmo de RL 156

Q-roulette: política de seleção baseada na função valor Q 59

RL: Reinforcement Learning (Aprendizado por Reforço) 149

RL-NFHB: Reinforcement Learning Neuro-Fuzzy Hierarchical
Binary Space Partitioning

RL-NFHP: Reinforcement Learning Neuro-Fuzzy Hierarchical Politree
(Reinforcement Learning - Neuro-Fuzzy Hierárquico Politree)

RL-NFHP $^{++}$: RL-NFHP utilizando os métodos de aceleração 106

RL-NFP: RL-Neuro-Fuzzy Politree $\quad 25$

$\begin{array}{ll}\text { RN: Redes Neurais } & 17\end{array}$

SARSA: algoritmo de RL State-Action-Reward-State-Action 155

$\begin{array}{ll}\text { SIF: Sistema de Inferência Fuzzy } & 17\end{array}$ 